J. Math. Kyoto Univ.

7-1 (1967) 93-112

\title{
Scattering for the Schrödinger operator in an exterior domain
}

\author{
By \\ Teruo IKEBE
}

\section{Introduction.}

This paper is a continuation of or supplement to [6], in which we have discussed the eigenfunction expansion problem connected with the Schrödinger operator $H_{q}=-\Delta+q$ in an exterior domain of $R^{3}$, where $q$ denotes the operator of multiplication by a function $q(x)$. More precisely, let $\partial \Omega$ be a bounded closed surface of class $C^{2}$ in $R^{3}, \Omega$ the (unbounded) domain exterior to $\partial \Omega$, and $\omega$ its (bounded) interior. Let $D$ be a dense linear subspace of $L_{2}(\Omega)$ characterized as follows :

$$
\begin{gathered}
D=\left\{f \in L_{2}(\Omega): f \in H_{2}(\Omega), \quad f \text { is continuous in } \bar{\Omega},\right. \\
\left.\left.\quad f\right|_{\text {ว } \Omega}=0\right\},
\end{gathered}
$$

where $\bar{\Omega}$ denotes the closure of $\Omega$ and $H_{2}(\Omega)$ is the totality of $L_{2}(\Omega)$-functions whose distribution derivatives up to second order are all in $L_{2}(\Omega)$. The formal differential operator $-\Delta+q$ equipped with $D$ as its domain of definition becomes a self-adjoint operator if $q(x)$ satisfies appropriate conditions as will be stated below. The operator thus obtained will be denoted by $H_{q}\left(D\left(H_{q}\right)\right.$ $\left.=D^{1)}\right)$, while the whole-space counterpart $\left(\Omega=R^{3}\right)$ of this operator with $q=0$ will be designated by $H^{0}$.

We have assumed in [6] and shall assume throughout also in the present paper that

1) The domain and range of an operator $A$ will be denoted by $D(A)$ and $R(A)$ respectively. 
(A)

$\left(q(x)\right.$ is a real-valued $L_{2}(\Omega)$-function locally Hölder-continu$\{$ ous in $\Omega$ except at a finite number of singularities, and satisfy the inequality $|q(x)| \leqslant C|x|^{-2-h}$ for $|x| \geqslant R_{0}$, with positive constants $C, h$ and $R_{0}$.

Results in the following few paragraphs are quoted from [6]. It has been shown that under the above assumption on $q(x)$ the spectrum of $H_{q}$ is divided into two parts: The whole positive real line is occupied by the absolutely continuous spectrum of $H_{q}$, i.e., $\left(E_{q}(\lambda) f, f\right)_{L_{2}(\Omega)}$ is, as a function of $\lambda$, absolutely continuous with respect to the ordinary Lebesgue measure for $\lambda \geqslant 0$, and for any $f \in L_{2}(\Omega)$, where $E_{q}(\lambda)$ is the right-continuous resolution of the identity associated with $H_{q}$;2) the negative part of the spectrum consists of discrete eigenvalues, i.e., isolated eigenvalues of finite multiplicity. We should remark, however, that the origin 0 is somewhat ambiguous; $E_{q}(\lambda)$ may or may not have a discontinuity at 0 , although this does not give rise to any complication in formulating the eigenfunction expansion theorem.

Now $H_{q}$ admits, besides $L_{2}(\Omega)$-eigenfunctions $\varphi_{q}{ }^{(n)}(n=1,2$, $\cdots, N ; N$ may be $\infty$ ) corresponding to the non-positive eigenvalues $\mu_{q}^{(n)}$, eigenfunctions $\varphi_{q}(x, k)$ associated with positive eigenvalues $|k|^{2}\left(k \in R^{3}, k \neq 0\right)$ such that, though not in $L_{2}(\Omega), \varphi_{q}(x, k)$ satisfies the Schrödinger equation

$$
-\Delta \varphi+q(x) \varphi=|k|^{2} \varphi \quad \text { in } \Omega
$$

as well as the boundary condition

$$
\left.\varphi\right|_{\partial \Omega}=0,
$$

and is bounded, continuous in $x \in \bar{\Omega}$ for each fixed $k \neq 0$. In terms of these eigenfunctions $\varphi_{q}^{(n)}$ and $\varphi_{q}(x, k)$, an arbitrary $f \in L_{2}(\Omega)$ can be expanded in the following sense: Let $Z_{q}$ and $Z_{q}{ }^{\prime}$ (the adjoint of $Z_{q}$ ) be defined by

2) The inner product and norm in a Hilbert space $\mathscr{H}$ are denoted by $(,)_{\mathscr{l}}$ and \|\|$\|_{\mathscr{H}}$ respectively. 


$$
\begin{array}{cc}
Z_{q} g(k)=(2 \pi)^{-3 / 2} 1 . \mathrm{i} . \mathrm{m} \cdot \int \varphi_{q}(x, k)^{*} g(x) d x & \left(g \in L_{2}(\Omega)\right),{ }^{3)} \\
Z_{q}^{\prime} g(x)=(2 \pi)^{-3 / 2} \text { 1.i.m. } \int \rho_{q}(x, k) g(k) d k & \left(g \in L_{2}\left(R^{3}\right)\right),
\end{array}
$$

and let

$$
g_{q}^{(n)}=\left(g, \varphi_{q}^{(n)}\right)_{L_{2}(\Omega)} \quad\left(g \in L_{2}(\Omega)\right) .
$$

Then all these definitions make sense, and $Z_{q}$ is isometric on $\left(1-E_{q}(0)\right) L_{2}(\Omega)$ to $L_{2}\left(R^{3}\right)^{4)}$ while the mapping: $g \rightarrow g_{q}{ }^{(n)}$ is unitary from $E_{q}(0) L_{2}(\Omega)$ onto $l_{2}\left(\operatorname{dim} l_{2}=N=\operatorname{dim} E_{q}(0) L_{2}(\Omega)\right)$, and we have

$$
\left\{\begin{array}{l}
f=Z_{q} Z_{q} f+\sum f_{q}^{(n)} \varphi_{q}^{(n)}, \\
f=Z_{q} Z_{q} f \quad\left(f \in\left(1-E_{q}(0)\right) L_{2}(\Omega)\right),
\end{array}\right.
$$

the summation being taken in the $L_{2}(\Omega)$-topology. Moreover, $Z_{q}$ maps onto $L_{2}\left(R^{3}\right)$ and hence $Z_{q}^{\prime}$ maps $L_{2}\left(R^{3}\right)$ isometrically onto $\left(1-E_{q}(0)\right) L_{2}(\Omega)$, and

$$
Z_{q} Z_{q}{ }^{\prime}=1
$$

[Otherwise, $Z_{q}^{\prime}$ is merely known to be a contraction from $L_{2}\left(R^{3}\right)$ onto $\left(1-E_{q}(0)\right) L_{2}(\Omega)$.] To the properties of $\left\{\varphi_{q}(x, k)\right\}$ that are displayed by the second equation of (1.7), and (1.8), we refer as completness and orthogonality, respectively.

In [6], however, we have remarked that the orthogonality of $\left\{\varphi_{q}(x, k)\right\}$ is not a simple consequence of the completeness that seems easier for us to handle, and that the orthogonality, which is, in fact, in a close relation with the unitary character of the scattering operator to be explained below, could be established with the aid of the time-dependent scattering theory. One of our purposes is to give a complete orthogonality proof along this line.

In passing it should be noticed that some of the notation

3) $(2 \pi)^{-3 / 2}$ is a normalization factor so that $Z_{q}$ be isometric, which entails the asymptotic behavior of $\varphi_{q}(x, k)$ being such that $\varphi_{q}(x, k)-\exp (i k \cdot x)=o(1)$ as $|x| \rightarrow \infty$. By $\lim \int \cdots$ is meant the limit in the mean for $R \rightarrow \infty$ of $\int_{B(0, R)} \cdots$ or $\int_{B(0, R) \cap \Omega} \cdots$, as the case may be, where $B(x, r)$ denotes the ball of radius $r$ about the point $x$. $z^{*}$ means the complex conjugate of $z$.

4) Irrespective of spaces under consideration, we denote by 1 the identity operator not distinguished in symbols from the numeral 1. 
introduced in this Introduction will be used in the sequel without further reference.

The scattering problem here consists in: Constructing the wave operators that relate the (system characterized by the) operator $H_{q}$ to the (system characterized by the) operator $H^{0}$ which has a very simple spectral structure; defining, in terms of the wave operators, the scattering operator that connects the asymptotic states of the $H_{q}$-system in the remote past and in the distant future, and proving that the scattering operator thus defined is unitary. In doing so, a little care must be taken in that we have to deal with operators acting in different Hilbert spaces; otherwise, the reasoning will not be essentially different from the one due to Jauch [7], Kato [8, Chap. 10] and Kuroda [9] among others. ${ }^{\text {) }}$

The problem presented above is also connected with the perturbation of continuous spectra of elliptic operators with variable boundaries and boundary conditions. For this we refer to Birman [1], where the invariance of absolutely continuous spectra is discussed in terms of what we have termed wave operators.

We should note here that there is a range of works on scattering theory related to the (hyperbolic) wave equation in exterior domains. The wave and scattering operators are defined in essentially the same manner as ours, though there may be noticed some seemingly different approaches. In this case, however, if $q(x)$ is present, various energy estimates will require a stronger restriction on $q(x)$. Eigenfunction expansions similar to ours can also be obtained in the framework of this theory. For this type of approach to scattering theory, we refer to Lax-Phillips [11], Schmidt [14], Shenk [15] and Thoe [16].

In $\S 2$ we shall prove a decay principle for wave packets that has a feature similar to the limiting amplitude principle and the energy decay for solutions of the wave or (time-dependent) Schrödinger equation (cf., e.g., Eidus [3], Ladyizhenskaya [10], Lax-Phillips [11], Morawetz [12], Odeh [13] and Zinnes [17]).

5) For the literature on scattering theory, Kato [8] may serve as a good reference. 
In $\S 3$ the existence of the wave operators will be shown (cf. Cook [2], Kato [8, Chap. 10] and Kuroda [9]). The remaining $\S \S 4$ and 5 are devoted to the proof that the scattering operator is unitary.

\section{$\S 2$. Decay of wave packets.}

Consider a physical system whose Hamiltonian is given by the Schrödinger operator $H_{q}$. The behavior of a wave packet in this system is described in terms of the unitary group $U_{q}(t)=$ $\exp \left(-i t H_{q}\right)$ associated with $H_{q}$. If the wave packet is initially in the state $f \in L_{2}(\Omega)$, then at an arbitrary time $t \in(-\infty, \infty)$, it will be given by $U_{q}(t) f$, which is a formal solution to the timedependent Schrödinger equation $i(d / d t) u=H_{q} u$, but not in the strict sense, because $U_{q}(t) f$ does not necessarily admit the application of $H_{q}$, unless $f \in D\left(H_{q}\right)$.

Now let $K$ be any bounded domain of $\bar{\Omega}$ and $\varepsilon(K)$ the projection defined by

$$
\mathcal{E}(K) f(x)=\left\{\begin{array}{ll}
f(x) & \text { if } x \in K \\
0 & \text { otherwise }
\end{array} \quad\left(f \in L_{2}(\Omega)\right) .\right.
$$

We shall prove the following decay principle for wave packets.

2. 1. Theorem. Let $f \in\left(1-E_{q}(0)\right) L_{2}(\Omega)$ and $K$ be a bounded domain of $\bar{\Omega}$. Then we have

$$
\lim _{t \rightarrow \pm \infty}\left\|\mathcal{E}(K) U_{q}(t) f\right\|_{L_{2}(\Omega)}=0 .
$$

Proof. It is sufficient to prove (2.2) for $f$ in a dense subset of $\left(1-E_{q}(0)\right) L_{2}(\Omega)$, for the norm of $\mathcal{E}(K) U_{q}(t)$ is uniformly bounded in $t \in(-\infty, \infty)$. We may assume, therefore, $f \in\left\{E_{q}\left(n^{2}\right)-\right.$ $\left.E_{q}\left(n^{-2}\right)\right) L_{2}(\Omega)$, where $n$ is a positive number. This implies

$$
Z_{q} f(k)=0 \quad \text { for }|k|>n^{6)}
$$

in view of [6], Theorem 7.1. Consequently, by the inversion and

6) Strictly speaking we have to add here the phrase "almost everywhere". In what follows we always omit the "almost every" or "almost everywhere", even when it be necessary in the strict sense of mathematical usage. 
diagonal representation formulas ([6], Theorem 7.1),

$$
U_{q}(t) f(x)=(2 \pi)^{-3 / 2} \int_{B(0, n)-B\left(0, n^{-1}\right)} \varphi_{q}(x, k) e^{-i t|k|^{2}} Z_{q} f(k) d k .
$$

Since for each fixed $x \in \bar{\Omega} \varphi_{q}(x, k)$ is continuous and hence bounded in $k \in B(0, n)-B\left(0, n^{-1}\right)$ ([6], Theorem 5.3), the Riemann-Lebesgue theorem can be applied to yield that (2.4) tends pointwise to 0 as $t$ tends to $\pm \infty$. On the other hand, since $\varphi_{q}(x, k)$ is bounded in $K \times\left(B(0, n)-B\left(0, n^{-1}\right)\right)([6]$, Theorem 5.3), we have

$$
\left|U_{q}(t) f(x)\right| \leqslant \text { const } \int_{B(0, n)-B(0, n-1)}\left|Z_{q} f(k)\right| d k \leqslant \text { const }\|f\|_{L_{2}(\Omega)}
$$

uniformly for $x \in K$, where the last constant may depend on $n$. Thus by the dominated convergence theorem (2.2) obtains. Q.E.D.

In 2. $1 \mathcal{E}(K)$ was the multiplicative operator by the characteristic function of $K$. Without any essential change of the proof we can replace $\mathcal{E}(K)$ by any multiplicative operator by a bounded function with bounded support, and thus obtain

2. 2. Theorem. Let $\varphi(x)$ be a bounded function with bounded support in $\bar{\Omega}$, and write $\varphi$ for the operator of multiplication by $\varphi(x)$. Then we have for $f \in\left(1-E_{q}(0)\right) L_{2}(\Omega)$

$$
\lim _{t \rightarrow \pm \infty}\left\|\varphi U_{q}(t) f\right\|_{L_{2}(\Omega)}=0 .
$$

2. 3. Remark. In the above proof no use has been made of the orthogonality of the eigenfunctions $\varphi_{q}(x, k)$ of $H_{q}$, i.e., the fact that $Z_{q}$ maps onto $L_{2}\left(R^{3}\right)$. (Cf. [6], Theorem 7.1 and remarks at the end of $\$ 7$.)

2. 4. Remark. The method of proof presented above applies also to the case where the exterior domain $\Omega$ is replaced by the whole space $R^{3}$. This, of course, entails some subsequent alterations in the formulation of the theorems, which, however, are more or less obvious.

\section{$\S 3$. Existence of the wave operators.}

This $\$$ gives a time-dependent treatment of the wave operators. The method is entirely independent of the eigenfunction expansion results for $H_{q}$, but is based upon some well-known results on 
Fourier transforms that, in fact, provide the eigenfunction expansion associated with $H^{0}\left(=-\Delta\right.$ in all of $\left.R^{3}\right)$.

3. 1. Definition. Let $\mathscr{P}: L_{2}\left(R^{3}\right) \rightarrow L_{2}(\Omega)$ be the "truncation" operator defined by

$$
(\mathscr{P} f)(x)=f(x) \quad(x \in \Omega) .
$$

Then the wave operators $W_{ \pm}\left(H_{q}, H^{0}\right): L_{2}\left(R^{3}\right) \rightarrow L_{2}(\Omega)$ are, if they exist,

$$
W_{ \pm}\left(H_{q}, H^{0}\right)=s-\lim _{t \rightarrow \pm \infty} W\left(t ; H_{q}, H^{0}\right),
$$

where $s$-lim means the strong limit being taken, and where

$$
W\left(t ; H_{q}, H^{0}\right)=e^{i t H_{q}} \mathscr{P} e^{-i t H^{0}} .
$$

3. 2. Theorem. The wave operators $W_{ \pm}\left(H_{q}, H^{0}\right)$ exist and are isometries defined on all of $L_{2}\left(R^{3}\right)$.

Proof. Let $\eta(x)$ be a smooth function defined on $\Omega$ which vanishes in a neighborhood of $\partial \Omega$ and assumes 1 outside a large ball $B(0, R)$. Then

$$
W\left(t ; H_{q}, H^{0}\right)=e^{i t H_{q}}(1-\eta) \mathscr{P} e^{-i t H^{0}}+e^{i t H_{q}} \eta \mathscr{P} e^{-i t H^{0}},
$$

where $\eta$ denotes the operator of multiplication by $\eta(x)$. In view of 2. 2 and 2.4 the first term on the right side of (3.4) can be seen to tend to 0 strongly as $t \rightarrow \pm \infty$, since $1-\eta(x)$ is a smooth function with compact support and $\exp \left(i t H_{q}\right)$ is unitary. Thus, in order to prove the existence of $W_{ \pm}\left(H_{q}, H^{0}\right)$ on all of $L_{2}\left(R^{3}\right)$, we need only show that the second term on the right of (3.4) converges. For this it is, in turn, sufficient to show the convergence on a dense subset $\mathscr{D}$ of $L_{2}(\Omega)$, because of the uniform boundedness in $t$ of the norm of $\exp \left(i t H_{q}\right) \eta \mathscr{P} \exp \left(-i t H^{0}\right)$.

Assuming $f \in D\left(H^{0}\right)$ we have

$$
\begin{aligned}
\frac{d}{d t}( & \left.e^{i t H_{q} \eta \mathscr{P}} e^{-i t H^{0}} f\right)=i e^{i t H_{q}}\left(H_{q} \eta \mathscr{P}-\eta \mathscr{P} H^{0}\right) e^{-i t H^{0}} f \\
= & -i e^{i t H_{q}} q(\Delta \eta) \mathscr{P} e^{-i t H^{0}} f-i e^{i t H} q(\operatorname{grad} \eta) \cdot \mathscr{P}\left(\operatorname{grad} e^{-i t H^{0}} f\right) \\
& +e^{i t H_{q}} q \eta \mathscr{P} e^{-i t H^{0}} f,
\end{aligned}
$$

because $\eta(x)$ vanishes identically near $\partial \Omega$, and hence $\eta(\Delta \mathscr{P} g)$ - 
$\eta \mathscr{P} \Delta g=0$ and $\operatorname{grad} \eta \cdot \operatorname{grad}(\mathscr{P} g)=\operatorname{grad} \eta \cdot \mathscr{P} \operatorname{grad} g$. Integrating (3.5) leads to

$$
\begin{aligned}
& \left\|e^{i t H_{q}} \eta \mathscr{P} e^{-i t H^{0}} f-\eta \mathscr{P} f\right\|_{L_{2}(\Omega)} \\
& \leqslant \int_{0}^{t}\left\|(\Delta \eta) \mathscr{P} e^{-i t H^{0}} f\right\|_{L_{2}(2)} d t \\
& +\int_{0}^{t}\left\|\operatorname{grad} \eta \cdot \mathscr{P} \operatorname{grad}\left(e^{-i t H^{0}} f\right)\right\|_{L_{2}(Q)} d t \\
& +\int_{0}^{t}\left\|q \eta \mathscr{P} e^{-i t H^{0}} f\right\|_{L_{2}(\mathcal{Q})} d t .
\end{aligned}
$$

Therefore, it suffices to show that

$$
\begin{aligned}
& \int_{-\infty}^{\infty} \|(\Delta \eta) \mathscr{P} e^{-i t H^{0} f \|_{L_{2}(\Omega)}} d t<\infty, \\
& \int_{-\infty}^{\infty}\left\|\operatorname{grad} \eta \cdot \mathscr{P} \operatorname{grad}\left(e^{-i t H^{0}} f\right)\right\|_{L_{2}(\Omega)} d t<\infty, \\
& \int_{-\infty}^{\infty} \| q \eta \mathscr{P} e^{-i t H^{0} f \|_{L_{2}(\Omega)} d t<\infty .}
\end{aligned}
$$

Now let $\mathscr{D}$ be the linear manifold spanned by the functions

$$
u_{a}(x)=\exp \left(-|x-a|^{2} / 2\right) \in L_{2}\left(R^{3}\right),
$$

where $a$ ranges over $R^{3}$. Clearly $\mathscr{D}$ is dense and $\subset D\left(H^{0}\right)$. Then we have ${ }^{\text {i) }}$

$$
e^{-i t H^{0}} u_{a}(x)=(1+2 i t)^{-3 / 2} \exp \left(-|x-a|^{2} /(2+4 i t)\right) .
$$

Consequently

$$
\left\|(\Delta \eta) \mathscr{P} e^{-i t H^{0}} u_{a}\right\|_{L_{2}(\Omega)} \leqslant\|\Delta \eta\|_{L_{2}(\Omega)}\left(1+4 t^{2}\right)^{-3 / 4} .
$$

$$
\left\|q \eta \mathscr{P} e^{-i t H^{0}} u_{a}\right\|_{\left.L_{2(Q)}\right)} \leqslant\|q\|_{L_{2}(\Omega)}\left(1+4 t^{2}\right)^{-3 / 4} .
$$

(3.12) and (3.13) yield (3.7) and (3.9) for $f \in \mathscr{D}$. In order to show (3.8) we compute the gradient of (3.11)

(3.14) $\operatorname{grad}\left(e^{-i t H^{0}} u_{a}\right)(x)=-(1+2 i t)^{-5 / 2}|x-a| \times$

$$
\times \exp \left(-|x-a|^{2} /(2+4 i t)\right) \operatorname{grad}|x-a|,
$$

which is seen to be bounded by $\left(1+4 t^{2}\right)^{-3 / 4}$. Hence

(3.15) $\left\|\operatorname{grad} \eta \cdot \mathscr{P} \operatorname{grad}\left(e^{-i t H^{0}} u_{a}\right)\right\|_{L_{2}(Q)} \leqslant\|\operatorname{grad} \eta\|_{L_{2}(\Omega)}\left(1+4 t^{2}\right)^{-3 / 4}$,

7) See Kato [8], p. 534. Cf. also Kuroda [9]. 
and (3.8) obtains for $f \in \mathscr{D}$. Since $\mathscr{D}$ is dense in $L_{2}\left(R^{3}\right)$, we have shown the existence of $W_{ \pm}\left(H_{q}, H^{0}\right)$ on all of $L_{2}\left(R^{3}\right)$.

It remains to show the isometry of $W_{ \pm}\left(H_{q}, H^{\circ}\right)$. One can write

$$
\begin{aligned}
\left\|W_{ \pm}\left(t ; H_{q}, H^{0}\right) f\right\|^{2}{ }_{L 2(\Omega)} & =\left\|\mathscr{P} e^{-i t H^{0} f}\right\|_{L_{2}(\Omega)}^{2} \\
& =\left\|e^{-i t H^{0}} f\right\|_{L_{2}\left(R^{3}\right)}^{2}-\left\|\mathcal{E}(\omega) e^{-i t H^{0}} f\right\|_{L_{2}\left(R^{3}\right)}^{2},
\end{aligned}
$$

where $\omega$ is the bounded domain interior to $\partial \Omega$, and where use has been made of the fact that $\mathcal{E}(\Omega)$ and $\mathcal{E}(\omega)$ are orthogonal.

From 2.4 and 2.1 it follows that the second term in the last member of (3.16) tends to 0 as $t \rightarrow \pm \infty$. The first term equals $\|f\|_{L_{2}\left(R^{3}\right)}^{2}$. This shows that $W_{ \pm}\left(H_{q}, H^{0}\right)$ are isometric. Q.E.D.

3. 3. Definition. Let $H=H_{0}(q(x)=0){ }^{8}{ }^{8}$ The wave operators $W_{ \pm}\left(H_{q}, H\right): L_{2}(\Omega) \rightarrow L_{2}(\Omega)$ are

$$
W_{ \pm}\left(H_{q}, H\right)=s-\lim _{t \rightarrow \pm \infty} W\left(t ; H_{q}, H\right),
$$

where

$$
W\left(t ; H_{q}, H\right)=e^{i t H} q e^{-i t H},
$$

if they exist.

The following theorem can be proved in exactly the same way as for the preceding theorem, if one puts $R^{3}=\Omega$ and $\mathscr{P}=1$.*)

3. 4. Theorem. $W_{i}\left(H_{q}, H\right)$ exist and are isometries on $L_{2}(\Omega)$.

We shall prove in the next $\S$ that $W_{ \pm}\left(H_{q}, H^{0}\right)$ and $W_{ \pm}\left(H_{q}, H\right)$ are complete in the following sense.

3. 5. Definition. $W_{ \pm}\left(H_{q}, H^{0}\right)$ and $W_{ \pm}\left(H_{q}, H\right)$ are complete if they map onto $\left(1-E_{q}(0)\right) L_{2}(\Omega)$.

3. 6. Theosem. Suppose $W_{ \pm}\left(H, H^{0}\right)$ and $W_{ \pm}\left(H_{q}, H\right)$ are complete. Then

$$
W_{ \pm}\left(H_{q}, H^{0}\right)=W_{ \pm}\left(H_{q}, H\right) W_{ \pm}\left(H, H^{0}\right),
$$

and $W_{ \pm}\left(H_{q}, H^{0}\right)$ are complete. (3.19) expresses a chain rule for the wave operators.

8) If $q(x)=0$, we agree to drop the subscript 0 . Thus, e.g., $E(\lambda)=E_{0}(\lambda)$

*) See the note added in proof at the end of this paper. 
Proof. (3.19) is obvious from

$$
e^{i t H_{q}} \mathscr{P} e^{-i t H^{0}}=\left(e^{i t H_{q}} e^{-i t H}\right)\left(e^{i t H} \mathscr{P} e^{-i t H^{0}}\right)
$$

and from the fact that the norm of each parenthesized operator on the right side of (3.20) is uniformly bounded in $t$. The completeness of $W_{ \pm}\left(H_{q}, H^{0}\right)$ readily follows if we note $E(0)=0$.

3. 7. Definition. Let $J$ denote the conjugation operator:

$$
J f(x)=f(x)^{*} \quad\left(f \in L_{2}\left(R^{3}\right) \text { or } \in L_{2}(\Omega)\right) .
$$

A self-adjoint operator $A$ is called real with respect to $J$ if

$$
J A=A J .
$$

$H^{0}$ and $H_{q}$ are clearly real with respect to $J$. Consequently, we have

$$
e^{-i t H^{0}}=J e^{i t H^{0}} J \quad \text { and } \quad e^{-i t H_{q}}=J e^{i t H_{q}} J,
$$

whence follows the following assertion.

3. 8. Theorem. The following relations hold:

$$
W_{+}\left(H_{q}, H^{0}\right)=J W_{-}\left(H_{q}, H^{0}\right) J, W_{+}\left(H_{q}, H\right)=J W_{-}\left(H_{q}, H\right) J .
$$

\section{\$4. The wave operators $\boldsymbol{W}_{ \pm}\left(\boldsymbol{H}, \boldsymbol{H}^{0}\right)$.}

In this $\S$ we shall make use of the results obtained in [6], $\S 7$ for $H(q(x)=0)$. The orthogonality of the eigenfunctions $\varphi(x, k)$, or the fact that $Z=Z_{0}$ maps onto $L_{2}\left(R^{3}\right)$, can be established without any use of the time-dependent scattering theory. With this we can proceed to define a "stationary" wave operator ( $U$ below) and show that it coincides with $W_{-}\left(H, H^{\circ}\right)$ that was defined in a time-dependent fashion in $\S 2$.

We first state the orthogonality theorem for $\{\varphi(x, k)\}$ :

4. 1. Theorem. The operator $Z: L_{2}(\Omega) \rightarrow L_{2}\left(R^{3}\right)$

$$
Z f(k)=(2 \pi)^{-3 / 2} \text { 1.i.m. } \int \varphi(x, k)^{*} f(x) d x
$$

maps onto $L_{2}\left(R^{3}\right)$.

Proof. See [5]. 
4. 2. Definition. Let $Z_{q}{ }^{\prime}$ be the adjoint (dual) of $Z_{q}$ :

$$
\begin{aligned}
\left(f, Z_{q}^{\prime} g\right)_{L_{2}(\Omega)}= & \left(Z_{q} f, g\right)_{L_{2}\left(R^{3}\right)} \\
& \left(f \in\left(1-E_{q}(0)\right) L_{2}(\Omega), g \in L_{2}\left(R^{3}\right)\right) .
\end{aligned}
$$

Since $Z_{q}$ is isometric on $\left(1-E_{q}(0)\right) L_{2}(\Omega)$ ([6], $\left.\S 7\right), Z_{q}^{\prime}$ is a contraction from $L_{2}\left(R^{3}\right)$ onto $\left(1-E_{q}(0)\right) L_{2}(\Omega)$, as is seen from the Ries $z$ theorem and the completeness of the eigenfunctions $\varphi(x, k)$ : $Z_{q}^{\prime} Z_{q}=1$ (see (1.7)). It is easy to see that $Z_{q}^{\prime}$ is expressible as

$$
Z_{q}^{\prime} f(x)=(2 \pi)^{-3 / 2} \text { 1.i.m. } \int \rho_{q}(x, k) f(k) d k \quad\left(f \in L_{2}\left(R^{3}\right)\right) .
$$

Let us also note that $Z^{\prime}=Z_{0}^{\prime}$ is unitary on $L_{2}\left(R^{3}\right)$ on to $L_{2}(\Omega)$ and inverse to $Z$, as is stated by 4.1 .

4. 3. Definition. The "stationary" wave operator $U: L_{2}\left(R^{3}\right)$ $\rightarrow L_{2}(\Omega)$ is defined to be

$$
U=Z^{\prime} \mathscr{F}=Z^{-1} \mathscr{F},
$$

where of is the Fourier transformation:

$$
\mathscr{F} f(k)=(2 \pi)^{-3 / 2} \text { 1.i.m. } \int e^{-i k \cdot x} f(x) d x .
$$

4. 4. Remark. $U$ is unitary on $L_{2}\left(R^{3}\right)$ onto $L_{2}(\Omega)$, as is clear from 4.1 and 4.2 .

4. 5. Theorem. $W_{-}\left(H, H^{0}\right)=U$, and hence $W_{-}\left(H, H^{0}\right)$ is complete.

Proof. Since the existence and isometry of $W_{-}\left(H, H^{0}\right)$ are known from 3.2, it is enough to show the weak convergence of $W(t)=W\left(t ; H, H^{0}\right)$ to $U$ for $t \rightarrow-\infty .^{9)} \quad$ Furthermore, we need only test the weak convergence on some dense subsets of $L_{2}\left(R^{3}\right)$ and $L_{2}(\Omega)$, respectively, because the norm of $W(t): L_{2}\left(R^{3}\right) \rightarrow L_{2}(\Omega)$ is uniformly bounded in $t$.

Let $D=\left\{f \in L_{2}\left(R^{3}\right): \mathscr{H} f \in C_{0}^{\infty}\left(R^{3}\right)\right\} \quad$ and $\quad D^{\prime}=\left\{g \in L_{2}(\Omega): Z g \in\right.$ $C^{\infty}(R)$ and has compact support exclusive of 0$\} . D$ and $D^{\prime}$ are dense in $L_{2}\left(R^{3}\right)$ and $L_{2}(\Omega)$ respectively.

9) In this case, however, the existence of $W_{-}\left(H, H^{0}\right)$ is a consequence of the proof that follows. For this see the remark, 4.6, after the proof. 
Now assuming $f \in D$ and $g \in D^{\prime}$, we have

$$
\begin{aligned}
(W(t) f, g)_{L_{2}(\Omega)} & =\left(\mathscr{P} e^{-i t H^{0}} f, e^{-i t H} g\right)_{L_{2}(\Omega)} \\
& =\int_{\Omega} \mathcal{F}^{-1} M(t) \mathscr{F} f(x)\left(Z^{\prime} M(t) Z g(x)\right)^{*} d x,
\end{aligned}
$$

where $M(t)$ denotes the multiplicative operator: $M(t) f(k)=$ $\exp \left(-i t|k|^{2}\right) f(k)$. Noting that the $\varphi(x, k)$, that may be viewed as the "kernel" of $Z$ ', is representable as

$$
\varphi(x, k)=e^{i k \cdot x}+h(x, k)
$$

(see [6], Theorem 3.2), we can rewrite (4.6) as follows :

$$
(W(t) f, g)_{L_{2}(\Omega)}=\left(\mathcal{F} f, Z_{g}\right)_{L_{2}\left(R^{3}\right)}+J_{1}+J_{2}+J_{3},
$$

where

$$
\begin{aligned}
& J_{1}=\int_{\omega}-\mathcal{F}^{-1} M(t) \mathscr{F} f(x)\left(\mathcal{F}^{-1} M(t) Z g(x)\right)^{*} d x . \\
& J_{2}=\int_{B(0, R) \cap \Omega} \mathcal{F}^{-1} M(t) \mathscr{F} f(x)\left[\int_{R^{3}} h(x, k) e^{-i t|k|^{2}} Z g(k) d k\right]^{*} d x, \\
& J_{3}=\int_{B(0, R)^{c}} \mathcal{F}^{-1} M(t) \mathscr{F} f(x)\left[\int_{R^{3}} h(x, k) e^{-i t|k|^{2}} Z g(k) d k\right]^{*} d x,{ }^{10)}
\end{aligned}
$$

$R$ being sufficiently large.

By using 2.1 and 2.4 $J_{1}$ is easily estimated to give

$$
J_{1} \rightarrow 0 \text { as } t \rightarrow-\infty \text {. }
$$

Similarly we get

$$
J_{2} \rightarrow 0 \quad \text { as } t \rightarrow-\infty \text {. }
$$

Now we are to estimate $J_{3}$. It can be seen from [6], $\$ 1$ and Theorem 3.2, that

$$
\begin{aligned}
h(x, k)= & \frac{1}{2 \pi} \int_{\partial \Omega} \frac{\partial}{\partial n_{y}} \frac{e^{i|k| \mid x-y_{\mid}}}{|x-y|} \sigma(y, k) d S_{y} \\
& -\frac{1}{4 \pi} \int_{\omega} \frac{e^{i|k||x-y|}}{|x-y|} \tau(y, k) d y,
\end{aligned}
$$

where $\sigma(y, k)$ and $\tau(y, k)$ are continuous on $\partial \Omega$ and $\bar{\omega}$ respectively, and are regular in $k$. By means of a straightforward computa-

10) $S^{c}$ denotes the complement of $S$. 
tion (cf. [6], Proposition 5.6 and [4], Lemma 3.2) using (4.12), we obtain

$$
h(x, k)=\frac{e^{i|k||x|}}{|x|} \eta_{1}(x, k)+\frac{1}{|x|^{2}} \eta_{2}(x, k)
$$

for $|x| \geqslant R$, where $R$ is sufficiently large, and where $\eta_{1}(x, k)$ and $\eta_{2}(x, k)$ are bounded in $x,|x| \geqslant R$, and regular in $k$, and $\partial \eta_{1}(x, k) / \partial|k|$ is bounded in $x,|x| \geqslant R$, if $|k|$ remains bounded by a constant.

The $k$-integral in $J_{3}$, where integration is actually performed over the finite region $\alpha \leqslant|k| \leqslant \beta$ on account of the assumption made on $g$, can now be written by $(4.13)$ as

$$
\begin{aligned}
& \frac{1}{|x|} \int_{\omega \leqslant|k| \leqslant \beta} e^{i|k||x|} \eta_{1}(x, k) e^{-i t|k|^{2}} Z g(k) d k \\
& \quad+\frac{1}{|x|^{2}} \int_{\omega \leqslant|k| \leqslant \beta} \eta_{2}(x, k) e^{-i t|k|^{2}} Z g(k) d k .
\end{aligned}
$$

For the first term of (4.14), by the above remarks on $\eta_{1}(x, k)$, we have

$$
\begin{gathered}
\text { 1st term of }(4.14)=\frac{1}{|x|}\left\{\left[\frac{e^{i|x| r-i t r^{2}}}{i(|x|-2 r t)} r^{2} \Phi(x, r)\right]_{\alpha}^{\beta}\right. \\
\left.-\int_{\infty}^{\beta} \frac{e^{i|x| r-i t r^{2}}}{i(|x|-2 r t)} \frac{\partial}{\partial r}\left[r^{2} \Phi(x, r)\right] d r\right\}
\end{gathered}
$$

with

$$
\Phi(x, r)=\int_{|\theta|=1} \eta_{1}(x, r \theta) Z_{g}(r \theta) d \theta \quad(k=r \theta),
$$

and hence

$$
\text { (4.17) } \quad \mid \text { st term of }(4.14) \mid \leqslant \frac{\text { const }}{(|x|-2 \alpha t)|x|} \leqslant \frac{\text { const }}{|x|^{2}}
$$

if $t$ is negative and large enough. The second term of (4.14) is easier to handle, and we obtain

$$
\mid 2 \text { nd term of }(4.14) \mid \leqslant \frac{\text { const }}{|x|^{2}} \text {. }
$$

Thus from (4.17), (4.18) and (4.9) we have as an estimate of $J_{3}$

$$
\left|J_{3}\right| \leqslant \text { const }\|f\|_{L_{2}\left(R^{3}\right)}\left(\int_{B(0, R)^{c}}|x|^{-4} d x\right)^{1 / 2},
$$

which can be made arbitrarily small by choosing $R$ sufficiently large. 
Now (4.8) together with (4.10), (4.11) and (4.19) gives

$$
(W(t) f, g)_{L_{2}(\Omega)} \rightarrow\left(\mathscr{F} f, Z^{\prime} g\right)_{L_{2}\left(R^{3}\right)}=(U f, g)_{L_{2}(\Omega)}
$$

for $t \rightarrow-\infty$, which was to be shown. Q.E.D.

4. 6. Remark. As has been noted in the course of the above proof, one does not have to have recourse to any time-dependent theory such as developed in 3.2 , in order to establish the existence and isometry of the wave operator $W_{-}\left(H, H^{0}\right)$. In fact, since $W(t)$ has been shown to converge weakly to $U$, and since $U$ is a surjective isometry, it follows that $\|f\|_{L_{2}\left(R^{3}\right)} \geqslant \lim \sup _{t \rightarrow-\infty}\|W(t) f\|_{L_{2}(\Omega)}$ $\geqslant\|U f\|_{L_{2}(\Omega)}=\|f\|_{L_{2}\left(R^{3}\right)}$. This is a well-known sufficient condition in order that weak convergence imply strong convergence. In consequence, the desired existence and isometry follow.

4. 7. Remark. The method of proof developed in 4.5 has utilized the fact that $\varphi(x, k)-\exp (i k \cdot x)$ is expressible in the from (4.13) when $|x|$ is large, with $\eta_{1}(x, k)$ and $\eta_{2}(x, k)$ having appropriate regularity in their arguments. Such an asymptotic expansion for the eigenfunctions is also possible for $H_{q}(q \neq 0)$, if $q(x)$ behaves asymptotically like $|x|^{-3-h}(h>0)$, as can be seen from [6], (5.26), Proposition 5.6 and Theorem 5.3. Also the analogue of 4.1 for $H_{q}$ holds good in this case. Thus we can develop a complete stationary method concerning the wave operators without resort to the results of $\$ 3$.

The following is an immediate consequence of 3.8 and 4.5.

4. 8. Theorem. $W_{+}\left(H, H^{0}\right)=J U J$, and $W_{-}\left(H, H^{0}\right)$ is complete.

\section{$\S 5$. The wave and scattering operator for $\boldsymbol{H}_{q}$.}

We have already discussed in $\S 3$ the existence of the wave operators $W_{ \pm}\left(H_{q}, H^{0}\right)$ (also $W_{ \pm}\left(H_{q}, H\right)$ ) by a time-dependent method. Now it remains to prove their completeness, which has been carried through in $\S 4$ for the special case of the wave operators $W_{ \pm}\left(H, H^{0}\right)$. Here we shall first be engaged in showing the completeness of $W_{ \pm}\left(H_{q}, H\right)$, and then proceed to that of $W_{ \pm}\left(H_{q}, H^{0}\right)$ making use of the chain rule 3.6. The reasoning will be almost 
a complete copy of [4], $\S 11$, and accordingly, some minute points of the discussion will be omitted.

It might be noted that it is not impossible to proceed directly with $W_{ \pm}\left(H_{q}, H^{0}\right)$ instead of $W_{ \pm}\left(H_{q}, H\right)$ without using the results of $\$ 4$, which, however, would lead to many a complication due to the fact that operators acting in different Hilbert spaces have to be dealt with at the same time.

5. 1. Definition. The "stationary" wave operators $U_{ \pm}\left(H_{q}, H\right)$ are defined as follows:

(5. 1) $U_{-}\left(H_{q}, H\right) f(x)=Z_{q}^{\prime} Z f(x)=(2 \pi)^{-3 / 2}$ 1.i.m. $\int \varphi_{q}(x, k) Z f(k) d k$,

(5. 2) $\quad U_{+}\left(H_{q}, H\right)=J U_{-}\left(H_{q}, H\right) J$.

At present we know only that $U_{ \pm}\left(H_{q}, H\right)$ are contraction operators on $L_{2}(\Omega)$ with range equal to $\left(1-E_{q}(0)\right) L_{2}(\Omega)$.

5. 2. Lemma. The adjoint $U_{-}\left(H_{q}, H\right)^{\prime}$ of $U_{-}\left(H_{q}, H\right)$ admits of a representation

$$
U_{-}\left(H_{q}, H\right)^{\prime} f(x)=Z^{\prime} Z_{q} f(x)=(2 \pi)^{-3 / 2} \text { l.i.m. } \int \varphi(x, k) Z_{q} f(k) d k,
$$

and the following relations hold:

$$
\begin{gathered}
U_{-}\left(H_{q}, H\right) U_{-}\left(H_{q}, H\right)^{\prime}=\left(1-E_{q}(0)\right), \\
\left\{\begin{array}{l}
U_{-}\left(H_{q}, H\right)^{\prime} F\left(H_{q}\right)=F(H) U_{-}\left(H_{q}, H\right)^{\prime}, \\
U_{-}\left(H_{q}, H\right)^{\prime} H_{q} \subset H U_{-}\left(H_{q}, H\right)^{\prime},
\end{array}\right.
\end{gathered}
$$

where $F(\lambda)$ is a bounded function and $F(H)\left(F\left(H_{q}\right)\right)$ is the operator defined in terms of $F(\lambda)$ and $E(\lambda)\left(E_{q}(\lambda)\right)$ in the oridinary sense of operational carculus.

Proof. (5.3) is immediate from (5.1). (5.4) is clear from (5.1) and (5.3). For the special case when $F(\lambda)$ is the characteristic function of $(-\infty, \mu]$, the first of (5.5) follows from

$$
U_{-}\left(H_{q}, H\right)^{\prime} E_{q}(\mu)=Z^{\prime} Z_{q} E_{q}(\mu)=Z^{\prime} \chi(\mu) Z_{q}
$$

and

$$
E(\mu) U_{-}\left(H_{q}, H\right)^{\prime}=Z^{\prime} \chi(\mu) Z Z^{\prime} Z_{q}=Z^{\prime} \chi(\mu) Z_{q}
$$


where $\chi(\mu)$ denotes the operator of multiplication by the characteristic function $\chi(k ; \mu)$ of $B(0, \sqrt{\mu})$ if $\mu \geqslant 0$, and 0 if $\mu<0$. The general $F(\lambda)$ case can be deduced from this. The second of (5.5) follows from the first. Q.E.D.

5. 3. Theorem. $W_{ \pm}\left(H_{q}, H\right)=U_{ \pm}\left(H_{q}, H\right)$, and hence $W_{ \pm}\left(H_{q}, H\right)$ are complete.

Proof. In virtue of (5.2) and 3.8 it is obviously sufficient to prove the assertion for the lower subscript. For the sake of simplicity let us omit $H$ and $H_{q}$ appearing in parentheses as "arguments" of $W_{ \pm}$and $U_{ \pm}$.

By 3.4 and (3.17)

$$
U_{-}^{\prime} W_{-}=s-\lim _{t \rightarrow-\infty} U_{-}^{\prime} W(t) .
$$

On the other hand, differentiation followed by integration of $U_{-}^{\prime} W(t)$ gives

$$
\begin{aligned}
A & =\left(U_{-}^{\prime} W_{-} f, g\right)_{L_{2}(\Omega)}-\left(U_{-}^{\prime} f, g\right)_{L_{2}(\Omega)} \\
& =i \int_{0}^{-\infty}\left(U_{-}^{\prime} e^{i t H} q q e^{-i t H} f, g\right)_{L_{2}(\Omega)} d t \\
& =i \lim _{\varepsilon} \downarrow_{0} \int_{0}^{-\infty} e^{\varepsilon t}\left(e^{i t H} U_{-}^{\prime} q e^{-i t H} f, g\right)_{L_{2}(\Omega)} d t,
\end{aligned}
$$

where $f \in D(H)=D\left(H_{q}\right)$ (see [6], Theorem 5.1) and $g \in L_{2}(\Omega)$, where we have used (5.5), and where the abelian limit has been taken by virtue of the fact that the (improper) $t$-integal exists, i.e., the fact that $W_{-}$exists (see 3.4).

For the integrand of the last member of (5.9) we have

$$
\begin{gathered}
\left(e^{i t H} U_{-}^{\prime} q e^{-i t H} f, g\right)_{L_{2}(\Omega)}=\left(U_{-}^{\prime} q e^{i t H} f, e^{-i t H} g\right)_{L_{2}(\Omega)} \\
=\left(Z U_{-}^{\prime} q e^{-i t H} f, Z e^{-i t H} g\right)_{L_{2}\left(R^{3}\right)} \\
=\left(Z_{q} q e^{-i t H} f, M(t) Z g\right)_{L_{2}\left(R^{3}\right)} \\
=(2 \pi)^{-3 / 2}\left(\int_{\Omega} \varphi_{q}(x, k)^{*} q(x) e^{-i t H+i t|k|^{2}} f(x), Z g(k)\right)_{L_{2}\left(R^{3}\right)},
\end{gathered}
$$

where $M(t)$ is the same as in the proof of 4.5 , and where the 1.i.m. in front of the integral has been left out, since $q \in L^{2}(\Omega)$.

Now substituting (5.10) in (5.9), we have 
(5. 11)

$$
\begin{aligned}
(2 \pi)^{3 / 2} A=i \lim _{\varepsilon \downarrow 0} \int_{R^{3}}\left[\int_{\Omega} \varphi_{q}(x, k)^{*} q(x)\left(\int_{0}^{-\infty} e^{-i t H+i t|k|^{2}+\varepsilon t} d t\right) f(x) d x\right] \times & \times Z g(k)^{*} d k \\
= & \lim _{\varepsilon \downarrow 0} \int_{R^{3}}\left[\int_{\Omega} \varphi_{q}(x . k)^{*} q(x)\left(H-|k|^{3}+i \varepsilon\right)^{-1} f(x) d x\right] Z g(k)^{*} d k \\
= & \lim _{\varepsilon \downarrow 0} \int_{R^{3}}\left[\int_{\Omega} f(x)\left(H-|k|^{2}-i \varepsilon\right)^{-1}\left(q(\cdot) \varphi_{q}(\cdot, k)\right)^{*}(x) d x\right] Z g(k)^{*} d k \\
= & \lim _{\varepsilon \downarrow 0} \int_{R^{3}}\left[\int_{\Omega} f(x)\left(\int_{\Omega} G\left(x, y, \sqrt{\left(|k|^{2}+i \varepsilon\right)}\right) q(y) \varphi_{q}(y, k) d y\right)^{*} d x\right] \times \\
= & \int_{R^{3}}\left[\int_{\Omega} f(x)\left(\int_{\Omega} G(x, y,|k|) q(y) \varphi_{q}(y, k)\right)^{*} d x\right] Z g(k)^{*} d k \\
= & \int_{R^{3}}\left[\int_{\Omega} f(x)\left(\varphi(x, k)-\varphi_{q}(x, k)\right)^{*} d x\right] Z g(k)^{*} d k \\
= & (2 \pi)^{3 / 2}\left[\left(Z f, Z_{g}\right)_{L_{2}\left(R^{3}\right)}-\left(Z_{q} f, Z_{g} g\right)_{L_{2}\left(R^{3}\right)}\right] \\
= & (2 \pi)^{3 / 2}\left[(f, g)_{L_{2}(\Omega)}-\left(U_{-}^{\prime} f, g\right)_{L 2(\Omega)}\right],
\end{aligned}
$$

where we have used the following: $\left(H-|k|^{2}-i \varepsilon\right)^{-1}$ is an integral operator of Carleman type with kernel $G\left(x, y, \sqrt{\left(|k|^{2}+i \varepsilon\right)}\right)$, i.e.,

$$
\left.\left(H-|k|^{2}-i \varepsilon\right)^{-1} f(x)=\int_{\Omega} G\left(x, y, \sqrt{\left(|k|^{2}+i \varepsilon\right)}\right) f\right) d y
$$

for $f \in L_{2}(\Omega)$ ([6], Theorem 2.1); the function

$$
\int_{\Omega} G(x, y, \kappa) q(y) u(y) d y
$$

where $u(x)$ is a bounded, continuous function of $x \in \bar{\Omega}$, is continuous in $x$ and $\kappa$ for $x \in \bar{\Omega}$ and $\operatorname{Im} \kappa \geqslant 0$ ([6], Propositions 5.1 and $5.10) ; \varphi_{q}(x, k)$ is continuous for $(x, k) \in \bar{\Omega} \times\left(R^{3}-\{0\}\right)$ and satisfies

$$
\varphi_{q}(x, k)=\varphi(x, k)-\int_{\Omega} G(x, y,|k|) q(y) \varphi_{q}(y, k) d y
$$

([6], (5.25), (5.26) and Theorem 5.2); $f$ and $Z g$ in (5.11) are assumed to be in $C_{0}^{\infty}(\Omega)$ and $C_{0}^{\infty}\left(R^{3}-\{0\}\right)$, respectively.

The facts enumerated above enable us to justify each step in (5.11) (for a more detailed argument, cf. [4], §11).

From (5.11) and the density in $L_{2}(\Omega)$ of $C_{0}^{\infty}(\Omega)$ and of $Z^{-1} C_{0}^{\infty}\left(R^{3}-\{0\}\right)$, it follows that 


$$
U_{-}^{\prime} W_{-}=1, \quad \text { or } \quad W_{-}=U_{-}
$$

as can be obtained by multiplying the first equation from left by

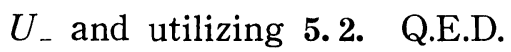

As a consequence of (5.15) one can see that $Z_{q}{ }^{\prime}$ is an isometry on $L_{2}\left(R^{3}\right)$ onto $\left(1-E_{q}(0)\right) L_{2}(\Omega)$. In fact, from (5.15), (5.1) and the isometry of $W_{-}\left(H_{q}, H\right)$ and $Z$ established in 3.4 and 4.1 , respectively, it follows that $Z_{q}{ }^{\prime}=W_{-}\left(H_{q}, H\right) Z^{-1}=W_{-}\left(H_{q}, H\right) Z^{\prime}$ is an isometry on $L_{2}\left(R^{3}\right)$ onto $\left(1-E_{q}(0)\right) L_{2}(\Omega)$, and thus one can give a proof of the orthogonality assertion in [6], Theorem 7.1:

5. 4. Theorem. $Z_{q}$ maps onto $L_{2}\left(R^{3}\right)$, or $\left\{\varphi_{q}(x, k)\right\}$ is an orthogonal family of eigenfunctions of $H_{q}$.

In view of the chain rule 3.6, 4.5 and 5.3 we have

5.5. Theorem. The wave operators $W_{ \pm}\left(H_{q}, H_{0}\right)$ are complete.

5. 6. Definition. The scattering operator $S_{q}: L_{2}\left(R^{3}\right) \rightarrow L_{2}\left(R^{3}\right)$ is defined to be

$$
S_{q}=W_{+}\left(H_{q}, H^{0}\right)^{\prime} W_{-}\left(H_{q}, H^{0}\right) .
$$

5. 7. Theorem. The scattering oporator $S_{q}$ is unitary on $L_{2}\left(R^{3}\right)$.

Proof. Direct from 5.5 and 5.6.

Now we shall touch upon the intertwining and commuting properties of $W_{ \pm}\left(H_{q}, H^{0}\right)$ and $S_{q}$ respectively.

5. 8. Theorem. The following relations hold:

$$
\begin{aligned}
W_{ \pm}\left(H_{q}, H^{0}\right) H^{0} & =H_{q} W_{ \pm}\left(H_{q}, H^{0}\right), \\
W_{+}\left(H_{q}, H_{0}\right)^{\prime} H_{q} & =H^{0} W_{ \pm}\left(H_{q}, H^{0}\right)^{\prime}, \\
S_{q} H^{0} & =H^{0} S_{q} .
\end{aligned}
$$

Proof. From 4.3, 4.5, 5.1, 5.3 and 3.6 one can see that

$$
W_{-}\left(H_{q}, H^{0}\right)=Z_{q}{ }^{\prime} Z Z^{-1} \mathscr{F}_{F}=Z_{q}^{\prime}{ }^{\prime} \text {. }
$$

Denote by $M$ the multiplicative operator: $M f(k)=|k|^{2} f(k)$. In view of [6], Theorem 7.1 (including the orthogonality assertion, 5. 4) 


$$
Z_{q}^{\prime} \mathcal{F}^{0} H^{0}=Z_{q}^{\prime} M^{\mathcal{F}} \mathcal{F}=H_{q} Z_{q}^{\prime} \mathcal{F}^{\prime},
$$

which proves (5.17) for the lower subscript. For the upper subscript (5.17) follows if one takes account of 3.8 .

(5.18) can be shown similarly. (5.19) is now an immediate consequence of (5.17), (5.18) and the definition of $S_{q}$. Q.E.D.

5. 9. Remark. So far we have been discussing with $H^{0}$ as the "reference" operator, in other words, the "reference" system has been the free-particle system in 3-space. Physically this is quite reasonable. Theoretically, however, the choice of the reference operator is quite arbitrary. One can thus take, for instance, $H$ as the reference operator. Then all the results so far obtained remain valid mutatis mutandis.

\section{REFERENCES}

[1] Birman, M. Sh.: Perturbation of continuous spectra of singular elliptic operators by the change of boundaries and boundary conditions. Vestnik Leningrad. Univ. No. 1, 22-55 (1962) (Russian).

[2] Cook, J. M.: Convergence to the Mфller wave-matrix. J. Mat. \& Phys. 36, 82-87 (1957).

[3] Eidus, D. M.: The principle of limiting absorption. Mat. Sb. (N.S.) 58 (100), 65-86 (1962) (Russian).

[4] Ikebe, T.: Eigenfunction expansion associated with the Schrödinger operators and their applications to scattering theory. Arch. Rational Mech. Anal. 5, 1-34 (1960).

[5] Ikebe, T.: Orthogonality of the eigenfunctions for the exterior poroblem connected with -4 . Arch. Rational Mech. Anal. 19, 71-73 (1965).

[6] Ikebe, T.: On the eigenfunction expansion connected with the exterior problem for the Schrödinger equation. Jap. J. Math. 36, 33-55 (1967).

[7] Jauch, J. M.: Theory of the scattering operator. Helv. Phys. Acta 31, 127-158 (1958).

[8] Kato, T.: Perturbation theory for linear operators. Berlin-Heidelberg-New York, Springer 1966.

[9] Kuroda, S. T.: On the existence and the unitary property of the scattering operator. Nuovo Cimento 12, 431-454 (1959).

[10] Ladyizhenskaya, O. A.: On the principle of limiting amplitude. Uspekhi Mat. Nauk 12, No. 3 (75), 161-164 (1957) (Russian).

[11] Lax, P. D. \& R. S. Phillips: Scattering theory. Bull. Am. Math. Soc. 70, 130142 (1964); Book with the same title. New York, Academic Press 1967.

[12] Morawetz, C. S. : Exponential decay of solutions of the wave equation. Comm. Pure Appl. Math. 19, 439 444 (1966).

[13] Odeh, F.: Principles of limiting absorption and limiting amplitude in scat. tering theory, I \& II. J. Mathematical Phys. 2, 794-800, 800-802 (1961). 
[14] Schmidt, G.: Scattering theory for Maxwell's equations in an exterior domain. (to appear).

[15] Shenk II, N. A.: Eigenfunction expansions and scattering theory for the wave equation in an exterior region. Arch. Rational Meth. Anal. 21, 120-150 (1966).

[16] Thoe, D.: Spectral theory for the wave equation with a potential term. Arch. Rational Mech. Anal. 22, 364-406 (1966).

[17] Zinnes, I. I.: Two theorems on scattering. Nuovo Cimento Ser X, 12, Suppl. No. 1, 87-99 (1959).

Note added in proof. The proof of 3.4 which asserts the existence and isometry of $W_{ \pm}\left(H_{q}, H\right)$ was incorrect. However, this may be proved as follows: By 4.5 the isometric $W_{ \pm}\left(H, H^{0}\right)$ exist and are complete, which implies the existence of $W_{ \pm}\left(H^{0}, H\right)$. Thus by means of the chain rule for wave operators, $W_{ \pm}\left(H_{q}, H\right)=W_{ \pm}\left(H_{q}, H^{0}\right) \times$ $W_{ \pm}\left(H^{0}, H\right)$ are known to exist and be isometric. 